Bitirme ve parlatma

sistemlerinin farklı tipteki

kompozit rezinlerin yüzey

pürüzlülüğüne etkisi

\section{Effect of different finishing and polishing systems on surface roughness of different types of resin composites}

\author{
Doç. Dr. Duygu Tuncer \\ Başkent Üniversitesi, Diş Hekimliği Fakültesi, \\ Restoratif Diş Tedavisi A.D., Ankara
}

\section{Dr. Derya Merve Halaçoğlu}

Yeditepe Üniversitesi, Diş Hekimliği Fakültesi, Restoratif Diş Tedavisi A.D., İstanbul

\section{Prof. Dr. Çiğdem Çelik}

Başkent Ủniversitesi, Diş Hekimliği Fakültesi,

Restoratif Diş Tedavisi A.D., Ankara

\section{Prof. Dr. Neslihan Arhun}

Başkent Üniversitesi, Diş Hekimliği Fakültesi, Restoratif Diş Tedavisi A.D., Ankara

Geliş Tarihi : 2 Mayıs 2016

Kabul tarihi: 29 Temmuz 2016

DOI: 10.5505/yeditepe.2016.43531

Yazışma Adresi:

Doç.Dr. Duygu Tuncer

Başkent Üniversitesi Diş Hekimliği Fakültesi, Restoratif Diş Tedavisi Anabilim Dalı

82.Sokak No:26 06490 - Bahçelievler / ANKARA

Telefon: (312) 2030000

E-posta: dtduygutuncer@gmail.com

\section{ÖZET}

Amaç: $\mathrm{Bu}$ in vitro çalışmanın amacı farklı bitirme ve parlatma sistemlerinin farklı tipteki kompozit rezinlerin yüzey pürüzlülüğü üzerine etkisini değerlendirmektir.

Gereç ve Yöntem: Bu çalışmada 3 farklı tipte kompozit rezin kullanılmıştır; Filtek Z250 (mikrohibrit kompozit, 3M ESPE), Filtek Z550 (nanohibrit kompozit, 3M ESPE), Aelite LS Posterior (düşük büzülmeli hibrit kompozit, Bisco). Toplam 144 adet disk şeklinde örnek hazırlandı ( $n=48)$. Örnekler, şeffaf bant altı yüzey (kontrol) ve uygulanan parlatma yöntemine göre rastgele 4 alt gruba ayrıldı ( $n=12)$ ve sırasıyla; Diamond Polish Mint (Ultradent), Soflex Disk (3M ESPE) ve OneGloss (Shofu) parlatma sistemleri uygulandı. Örneklerin yüzey pürüzlülüğü; yüzey pürüzlülük ölçüm aleti (Mitutoyo Surftest SJ-201) kullanılarak ölçüldü. Elde edilen veriler istatistiksel olarak analiz edildi $(p<0.05)$.

Bulgular: Bütün gruplarda şeffaf bant altı yüzey (kontrol) en pürüzsüz olarak ölçülmüş ve materyaller arasında farklılık görülmemiştir. Filtek Z250 ve Filtek Z550 gruplarında Diamond Polish ya da Soflex disk kullanıldığında benzer pürüzlülük değerleri elde edilirken, Onegloss daha yüksek pürüzlülük değerleri göstermiştir $(p<0.05)$. Aelite grubunda ise Soflex ile diğer yöntemlere göre daha pürüzsüz yüzey elde edilmiştir $(p<0.05)$. Bununla birlikte, kontrol grubu dışında, Aelite'ın diğer restoratif materyallerden daha pürüzlü yüzeye sahip olduğu bulunmuştur $(p<0.05)$.

Sonuç: Kompozit rezin tipinden bağımsız olarak alüminyum oksit disk sistemi ile en az pürüzlü yüzey elde edilmiştir.

Anahtar kelimeler: Kompozit rezin, polisaj sistemleri, yüzey pürüzlülüğü.

\section{SUMMARY}

Aim: The aim of this in vitro study was to evaluate the effects of different finishing and polishing systems on surface roughness of different types of resin composites.

Materials and methods: In this study, 3 types of resin composites were used; Filtek Z250 (microhybrid resin composite, 3M ESPE), Filtek Z550 (nanohybrid resin composite, 3M ESPE), Aelite LS Posterior (low-shrinkage hybrid resin composite, Bisco). A total of 144 disc shaped specimens were prepared $(n=48)$. Specimens were randomly divided into 4 subgroups according to finishing and polishing systems and Mylar strip as 
control ( $n=12)$ and Diamond Polish Mint (Ultradent), Soflex Disk (3M ESPE) and OneGloss (Shofu) finishing and polishing systems used, respectively. The surface roughness values were determined with a profilometer (Mitutoyo Surftest SJ-201). Data were statistically analyzed $(p<0.05)$.

Results: The Mylar strip produced the smoothest surface in all groups and there was no significant difference between the restorative materials. In Filtek Z250 and Filtek Z550 groups, when Diamond Polish or Soflex disk used, similar surface roughness values were measured, Onegloss exhibited higher roughness values $(p<0.05)$. In Aelite group, Soflex produced smoother surface than the other techniques $(p<0.05)$. Additionally, Aelite exhibited rougher surfaces than the other restorative materials except control group $(p<0.05)$.

Conclusion: Aluminum oxide disks system produced the smoothest surface regardless of the resin composite used.

Key words: Composite resin, polishing systems, surface roughness.

\section{GíRiş}

Yeni estetik restoratif materyallerdeki hızı gelişmeler ile ilişkili olarak estetik diş hekimliğine talep giderek artmaktadır. Bununla birlikte, adeziv sistemlerdeki formulasyonların gelişmesi ve uygulama kolaylığı sağlanması gibi etkenler de kompozit rezinlerin kullanım oranlarını arttırmaktadır. ${ }^{2}$ Kompozit rezinler; doldurucu tipi, doldurucu oranı, doldurucu partikül büyüklüğü ve materyallerin fiziksel ve mekanik özelliklerine göre sınıflandırılabilirler. ${ }^{3}$ Nanoteknolojideki gelişmeler ile birlikte, bu teknolojinin kullanıldığı nanokompozit rezinler üretilmiştir. ${ }^{4}$ Nanokompozitlerin, geliştirilmiş mekanik ve optik özelliklerinin yanı sıra, geleneksel hibrit ve mikrohibrit kompozitlere göre daha iyi polisajlanabilme özelliğine ve artmış aşınma direncine sahip oldukları bildirilmiştir. ${ }^{5}$

Bu nedenle, diş rengindeki restoratif materyallerin estetik ve uzun ömürlü olmaları, restorasyona uygulanan bitirme ve polisaj işlemlerinin iyi bir şekilde yapılmış olmasına sıklıkla bağlıdır. ${ }^{6,7}$ Yüksek kalitedeki bitirme ve polisaj işlemi restorasyonun başarısını arttıır, başarısız bitirme ve polisaj ile elde edilen yüzey pürüzlüdür, renklenmeye açıktır, plak birikimi dişetinde irritasyon, restorasyonda kenar renklenmesine ve sekonder çürüğe neden olur. ${ }^{8}$

Restoratif diş hekimliği uygulamalarının en önemli amacı diş dokusuna fiziksel özellikleri en yakın olan restorasyonlar uygulamaktır. Yüzey pürüzlülüğü, parlaklık ve renk uyumu bu özelliklerden birkaçıdır. Bitirme ve parlatma işlemleri, materyalin kendi fizikokimyasal özelliklerinin yanında, plak birikimi ve renklenmeyi etkileyebilir. ${ }^{9}$ İyi bitirme ve parlatma yapılmış restorasyonların mine dokusuna benzer yüzeye sahip olması amaçlanmaktadır. ${ }^{10}$ Pürüzsüz bir yüzey elde etmek için piyasada çok çeşitli bitirme ve parlatma sistemi bulunmaktadır.11,12 Bununla birlikte, kompozit rezin materyallerdeki doldurucu partikül boyutu ve tipi de bitirme ve parlatma işlemlerinin başarısını etkilemektedir.

$\mathrm{Bu}$ in vitro çalışmanın amacı; farklı polisaj sistemlerinin; bir mikrohibrit, bir nanohibrit ve bir posterior kompozitin yüzey pürüzlülüğü üzerine etkisini değerlendirmektir.

\section{GEREÇ VE YÖNTEM}

Bu çalışmada 3 farklı tipte kompozit rezin kullanılış̧ır: Filtek Z250 (mikrohibrit kompozit, 3M ESPE, St.Paul, MN, ABD), Filtek Z550 (nanohibrit kompozit, 3M ESPE, St.Paul, MN, ABD), Aelite LS Posterior (posterior kompozit, Bisco, Schamburg, ABD) (Tablo 1). Her bir kompozit rezinden 48 adet olmak üzere toplam 144 adet disk şeklinde örnek 7x2 $\mathrm{mm}$ boyutlarındaki teflon kalıplar içinde, alt ve üst kısmında pürüzsüz bir yüzey elde etmek için, şeffaf matriks bandı yerleştirilerek hazırlandı. Kompozit rezin, kalıp içine tek tabaka halinde, kalıp içinde boşluk kalmayacak şekilde el aletleriyle yerleştirildi. Kompozit rezin yüzeyine şeffaf matriks band yerleştirilip, ince cam lamel ile basınç uygulanarak, yoğunluğu $1000 \mathrm{~mW} / \mathrm{cm}^{2}$ olan LED ışık cihazı kullanılarak 20 sn ışıkla polimerize edildi (Hilux LEDMAX-550, Benlioğlu Dental Ankara, Türkiye). Örneklere alt yüzeylerinden de $20 \mathrm{sn}$ ışık uygulandı. Örnekler $37^{\circ} \mathrm{C}$ 'de distile su içerisinde 24 saat süre ile bekletildi. Örnekler, şeffaf bant altı yüzey (kontrol) ve uygulanan parlatma yöntemine göre 4 alt gruba ayrıldı $(n=12)$. Her gruptaki örnekler numaralandırıldı. Kullanılan 
polisaj sistemleri Tablo 1'de belirtildi.

Örneklerin bitirme işlemleri 1200 gritlik silikon karbid zımparalar kullanılarak su altında yapıldı, daha sonra sırasıyla Diamond Polish Mint (Ultradent Products, South Jordan, UT, ABD), Soflex Diskler (3M ESPE, St.Paul, MN, ABD) ve OneGloss (Shofu Inc., Kyoto, Japonya) ile üretici firmasının önerileri doğrultusunda parlatıldı. Bütün gruplarda, parlatma işlemi düşük turlu alet kullanarak, su soğutması altında yapıldı. Her 5 örnekte bir yeni bir lastik ya da disk kullanıldı. Bütün örnekler $37^{\circ} \mathrm{C}$ distile suda 24 saat bekletildi.

Örneklerin pürüzlülük ölçümleri, yüzey pürüzlülük ölçüm aleti kullanılarak (Mitutoyo Surftest SJ-<, 201, ABD) (cut off değeri=0.8 mm) $4 \mathrm{~mm}$ aralıklarda ve cihazın ucu $2 \mathrm{~mm} / \mathrm{sn}$ hızla ilerletilerek 3 farklı yerden ölçüldü ve orta hattan profile uzaklık değerlerinin aritmetik ortalaması alınarak ortalama yüzey pürüzlülüğü (Ra,ųm) değerleri elde edildi.

İstatistiksel analizler IBM SPSS Versiyon 21 kullanılarak yapıldı. Veriler Tekrarlı Ölçümlerde Varyans Analizi kullanılarak değerlendirildi. İkili karşılaştırmalar için de Bonferroni Testi kullanıldı $(\mathrm{p}<0.05)$.

\begin{tabular}{|c|c|c|c|}
\hline Materyal & Materyal Tipi & Özellikler & $\begin{array}{c}\text { Üretici } \\
\text { Firma }\end{array}$ \\
\hline Filtek Z250 & $\begin{array}{l}\text { Mikrohibrit } \\
\text { Kompozit }\end{array}$ & $\begin{array}{l}\text { Bis-GMA, Bis-EMA, UDMA, } \\
\text { foto-başlatıclar ve stabilize } \\
\text { ediciler Doldurucu } \\
\text { Zirkonyum/silika doldurucu } \\
{[0.01-3.5 \mathrm{~mm}]: 84.5 \mathrm{wt} \% \text {, }} \\
60 \mathrm{vol} \%\end{array}$ & $\begin{array}{l}\text { 3M ESPE, } \\
\text { St. Paul, } \\
\text { MN, ABD }\end{array}$ \\
\hline Filtek Z550 & $\begin{array}{l}\text { Nanohibrit } \\
\text { Kompozit }\end{array}$ & $\begin{array}{l}\text { Silane processed ceramic, } \\
\text { silane processed silica, BIS- } \\
\text { GMA, UDMA, bısphenol a } \\
\text { polyethylene glycol diether } \\
\text { dımethacrylate, TEGDMA }\end{array}$ & $\begin{array}{l}\text { 3M ESPE, } \\
\text { St. Paul, } \\
\text { MN, ABD }\end{array}$ \\
\hline $\begin{array}{l}\text { Aelite LS Pos- } \\
\text { terior }\end{array}$ & $\begin{array}{l}\text { Düşük büzülm- } \\
\text { eye sahip hibrit } \\
\text { kompozit }\end{array}$ & $\begin{array}{l}\text { Bis-EMA, TEGDMA glass } \\
\text { frit, amorphous silica, filler } \\
\text { vol } 74 \% \text {, filler weight } 88 \%\end{array}$ & $\begin{array}{l}\text { Bisco, Inc, } \\
\text { Scham- } \\
\text { burg, IL, } \\
\text { ABD }\end{array}$ \\
\hline $\begin{array}{l}\text { Diamond } \\
\text { Polish }\end{array}$ & $\begin{array}{l}\text { Elmas Polisaj } \\
\text { Patı }\end{array}$ & $\begin{array}{l}\text { Nane aromalı, beyaz, } 1.0 u \text { m } \\
\text { ve } 0.5 \text { ųm iki farklı gren, } \\
\text { su içine emdirilmiş elmas } \\
\text { partiküllerden oluşur. }\end{array}$ & $\begin{array}{l}\text { Ultradnt } \\
\text { Products,- } \\
\text { South } \\
\text { Jordan, } \\
\text { Utah, ABD }\end{array}$ \\
\hline Soflex Disk & Polisaj Disk Seti & $\begin{array}{l}\text { Aluminium oxide coated } \\
\text { discs (coarse, medium, } \\
\text { fine, super fine) } / 15 \text { s for } \\
\text { each disc }\end{array}$ & $\begin{array}{l}\text { 3M ESPE, } \\
\text { St. Paul, } \\
\text { MN, ABD }\end{array}$ \\
\hline One Gloss & $\begin{array}{l}\text { Tek aşamalı } \\
\text { bitirme ve } \\
\text { polisaj seti }\end{array}$ & $\begin{array}{l}\text { Synthetic rubber (poly- } \\
\text { vinylsiloxiane) Abrasive } \\
\text { grain (Al2O3) Silicon oxide } \\
\text { (SiO2). }\end{array}$ & $\begin{array}{l}\text { Shofu Inc., } \\
\text { Kyoto, } \\
\text { JAPONYA }\end{array}$ \\
\hline
\end{tabular}

Tablo 1: Çalışmada kullanılan materyaller, içerikleri ve üretici firmaları

\section{BULGULAR}

Tablo 2'de kompozit rezin gruplarının parlatma sonrasında ölçülen ortalama Ra değerleri görülmektedir. Bütün gruplarda şeffaf bant altı yüzey (kontrol) istatistiksel olarak anlamlı olacak şekilde en pürüzsüz olarak ölçülmüş ve materyaller arasında farklılık görülmemiştir. Filtek Z250 ve Filtek Z550 gruplarında Diamond Polish ya da Soflex disk kullanıldığında benzer pürüzlülük değerleri elde edilirken, Onegloss daha yüksek değerler göstermiştir(p<0.001). Aelite grubunda ise polisaj yöntemlerinden Soflex ile diğer yöntemlere göre daha pürüzsüz yüzey elde edilmiştir $(p<0.001)$. Materyaller arasındaki istatistiksel farklılıklar değerlendirildiğinde ise kontrol grubu dışında tüm yöntemlerde Aelite grupları diğerlerinden anlamlı olarak farklı bulunmuştur $(p<0.001)$.

\begin{tabular}{|l|l|l|l|l|}
\hline Ra Değerleri & Filtek Z250 & Filtek Z550 & Aelite LS & P değerleri \\
\hline Mylar (Kontrol) & $0.75 \pm 0.018$ & $0.084 \pm 0.02$ & $0.123 \pm 0.019$ & 0.099 \\
\hline $\begin{array}{l}\text { Diamond } \\
\text { Polish }\end{array}$ & $0.169 \pm 0.048$ & $0.18 \pm 0.066$ & $0.387 \pm 0.057$ & $<0.001$ \\
\hline Soflex Disc & $0.153 \pm 0.028$ & $0.16 \pm 0.036$ & $0.268 \pm 0.06$ & $<0.001$ \\
\hline One Gloss & $0.283 \pm 0.079$ & $0.339 \pm 0.105$ & $0.411 \pm 0.074$ & $<0.001$ \\
\hline P değerleri & $<0.001$ & $<0.001$ & $<0.001$ & \\
\hline
\end{tabular}

Tablo 2: Grupların yüzey pürüzlülüğü değerleri (Ra,ų) (Ortalama SS)

\section{TARTIŞMA}

Kompozit rezinlerin bitirme ve parlatma işlemleri; estetik beklentilerin karşılanması ve restorasyonun ömrü açısından kritik bir öneme sahiptir. Bu in vitro çalışma farklı tipteki kompozit rezinlere, farklı özelliklere sahip parlatma sistemleri uygulandığında elde edilen pürüzlülük değerlerini incelemiştir.

Bu çalışmada, klinik olarak yapılan bitirme işlemini taklit etmek ve standardizasyon sağlamak için, bitirme işlemi 1200 gridlik silikon karbid zımpara kullanılarak akan su altında yapılmıştı. ${ }^{13}$ Yapılan çalışmalarda şeffaf matriks bant altında polimerize edilen kompozit yüzeylerinin en az pürüzlülük değerlerine sahip olduğu bildirilmiştir. ${ }^{14-16} \mathrm{Bu}$ çalışmada da benzer sonuçlar elde edilmiştir. Bununla birlikte, şeffaf bant altında elde edilmiş kompozit rezin yüzeyinde organik içeriğin fazla olması, bu tabakanın bitirme ve parlatma işlemi ile uzaklaştırılmasını gerektirmektedir. Bu şekilde daha sert ve aşınmaya karşı daha dirençli bir kompozit yüzeyi elde edilir. $^{17,18}$ 
Abraziv disk setleri kullanıldığında elde edilen kompozit rezin yüzeylerinin diğer yöntemlere göre daha pürüzsüz olduğu bildirilmiştir. ${ }^{19}$ Parlatma için en iyi yöntemin abraziv diskler olduğu belirtilmiştir. ${ }^{7,20-23}$ Bu çalışmada, diğer çalışmalara benzer olarak her üç kompozit rezin yüzeyi Soflex disklerle parlatıldığında, diğer sistemlere göre daha pürüzsüz yüzeyler elde edilmiştir.

Parlatma sistemleri birbiriyle karşılaştırıldığında Filtek Z250 ve Z550 gruplarında Soflex ve Diamond polish sistemleri arasında istatistiksel olarak farklılık gözlenmezken, Aelite LS Posterior grubunda Onegloss ve Diamond Polish yüzey pürüzlülüğünde artışa neden olmuş, Soflex en iyi yöntem bulunmuştur. Bitirme/parlatma sisteminin etkinliği, materyale (doldurucu boyutu, oranı), kullanılan aşındırıcının tipine, abrazivin uygulanma süresine, uygulanan basınca, abrazivin şekline göre farklılık gösterebilir. ${ }^{17}$ Elmas partikülleri her zaman alüminadan daha serttir; böylece kompozit yüzeyinde daha fazla çiziğe neden olabilir ki bu da daha pürüzlü bir yüzey demektir. ${ }^{24} \mathrm{Bu}$ çalışmada kullanılan Soflex diskler aluminyumoksit, Diamondpolish elmas partiküller ve OneGloss aluminyumoksit ve silikonoksit içermektedir. Ayrıca alüminyumoksit partikülleri kompozit yüzeyinde elde edilen pürüzsüz yüzeyi partiküller ve organik matriksten eşit miktarda uzaklaştırmasıyla sağlar. Ancak bu sistemin bir dezavantajı vardır; özellikle posterior bölgedeki konturlu ve girintili yüzeylerin ve anatomik detayların polisajının yapılmasında disklerin kullanılması biraz zor olmaktadır.?

Klinik uygulama zamanını azaltmak için, son yıllarda iki ve tek aşamalı polisaj sistemleri geliştirilmiştir. Tek aşamalı polisaj sistemlerindeki azaltılmış uygulama aşamaları ve zamanı ve ayrıca çapraz enfeksiyon riskinin daha az olması tek aşamalı polisaj sistemlerinin tercih edilmesini sağlar. ${ }^{25,26}$ Yap ve ark. ${ }^{27}$ ve St-Georges ve ark. ${ }^{28}$ tek aşamalı polisaj sistemlerinin, çok aşamalı polisaj sistemleriyle benzer sonuçlar verdiğini, harcanan zamanı ve maliyeti azaltmak için tek aşamalı sistemlerin tercih edilebileceğini bildirmişlerdir. Geçmiş yıllarda bu konu ile ilgili yapılan çalışmalarda farklı sonuçlar elde edilmiştir. Bizim çalışmamıza benzer olarak, Özel ve ark. ${ }^{29}$ farklı polisaj sistemlerinin kompozitlerin yüzey pürüzlülüğüne et- kisini inceledikleri çalışmalarında tek aşamalı polisaj sistemi olan OneGloss'un kullanılan diğer sisteme ve Soflex disklere göre daha pürüzlü yüzeye neden olduğunu bulmuşlardır. Başka çalışmalarda tek aşamalı Po-Go ve çok aşamalı Soflex diskler benzer pürüzlülük değerleri göstermiştir. ${ }^{12,30}$ Bununla beraber çok aşamalı sistemlerin daha iyi olduğunu belirten sonuçlar da vardır. ${ }^{31-33}$

Bu çalışmada, kontrol grubu haricinde Aelite LS posterior örnekler, daha pürüzlü yüzeylere sahip olmuştur. Yalnızca Onegloss ile polisajlandığında FiltekZ250, Aelite LS Posterior'a göre daha pürüzsüz değerler göstermiştir. Yüzey pürüzlülüğü, doldurucu partiküllerin yüzeydeki oranı, sertliği, boyutu, monomer yapısı, matriks/partikül ilişkisi gibi kompozit yapısıyla ilgili birçok faktörden etkilenmektedir. ${ }^{34}$ Daha sert ve büyük doldurucu partiküller içeren kompozitler, bitirme/ parlatma sonrasında daha yüksek Ra değerleri gösterirler. ${ }^{20,23,31,35}$ Aelite LS Posterior doldurucu miktarı, yapısı, doldurucu oranı nedeniyle diğer kompozit rezinlere göre polisaj sonrasında daha pürüzlü bir yüzey göstermiş olabilir. Bir çalışmada polisaj sonrasında minifil-hibrit kompozitin (Esthet-X), tepilebilir (Solitaire II) kompozitten daha düzgün bir yüzeye sahip olduğunu bildirilmiştir. ${ }^{36}$ Yapılan bazı çalışmalarda, farklı kompozitlerin yüzey pürüzlülüğüne polisaj sistemlerinin etkisini değerlendirdikleri çalışmalarında materyaller arasında fark oluşmadığı bildirilmiştir.12,13,37 Bir çalışmada nanofil ve nanohibrit kompozitlerin yüzey pürüzlülüğüne polisaj sistemlerinin etkisi değerlendirilmiş, yüzey pürüzlülüğünün kullanılan polisaj sistemine göre farklıık yarattığı belirtilmiştir. ${ }^{38}$ Diğer bir çalışmada tek ve çok aşamalı polisaj sistemlerinin farklı kompozitlerin yüzey pürüzlülüğüne etkisinin materyale bağımlı olduğu belirtilmiştir. ${ }^{25}$ Aynı şekilde başka bir çalışmada yüzey pürüzlülüğünün kullanılan materyal içeriği ve polisaj sistemine göre farklılık gösterdiği bildirilmiştir. ${ }^{39} \mathrm{Bu}$ çalışmada da kullanılan polisaj sistemine göre kompozit rezinler farklı pürüzlülük değerleri göstermiştir.

Bu çalışmada en yüksek elde edilen ortalama pürüzlülük değeri 0.41 olarak belirlenmiştir. Bu değer klinik olarak kabul edilebilir sınırlardadır. Chung ${ }^{36} 1$ ųm' den az yüzey 
pürüzlülüğüne sahip restorasyonların klinik şartlarda kabul edilebilir olduğunu bildirmiştir.

Profilometre in vitro olarak yapılan yüzey pürüzlülüğü çalışmalarında sıkıkla kullanılmaktadır. ${ }^{15,35,40}$ Profilometre ile iki boyutlu veri elde edilebilir. Ancak yüzeyin karmaşık yapısını analiz etmek için daha ayrıntılı atomik kuvvet mikroskobu ve taramalı elektron mikroskobu kombinasyonu kullanılarak yapılabilir. ${ }^{30} \mathrm{Bu}$ çalışmada pürüzlülük ölçümü yalnızca profilometre kullanılarak yapılmış olması bir kısıtlıık olarak düşünülmektedir. Bu nedenle ileriki çalışmalarda yüzey pürüzlülüğünün değerlendirmesinde farklı yöntemlerin birlikte kullanılması planlanmaktadır

\section{SONUÇ}

Yapılan bu in vitro çalışmanın kısıtlııkları dahilinde, uygulanan kompozit rezin tipinden bağımsız olarak diskle yapılan parlatma işlemlerinden sonra en az pürüzlü yüzey elde edilmiştir.

\section{KAYNAKLAR}

1. Gaintantzopoulou M, Kakaboura A, Vougiouklakis G. Colour stability of tooth-coloured restorative materials. Eur $\mathrm{J}$ Prosthodont Restor Dent 2005; 13: 51-56.

2. Sarac D, Sarac YS, Kulunk S, Ural C, Kulunk T. The effect of polishing techniques on the surface roughness and color change of composite resins. J Prosthet Dent 2006; 96: 3340.

3. Senawongse $P$, Pongprueksa P. Surface roughness of nanofill and nanohybrid resin composites after polishing and brushing. J Esthet Restor Dent 2007; 19: 265-273; discussion 74-75.

4. Ure D, Harris J. Nanotechnology in dentistry: reduction to practice. Dent Update 2003; 30: 10-15.

5. Mitra SB, Wu D, Holmes BN. An application of nanotechnology in advanced dental materials. J Am Dent Assoc 2003; 134: 1382-1390.

6. Reis AF, Giannini M, Lovadino JR, Ambrosano GM. Effects of various finishing systems on the surface roughness and staining susceptibility of packable composite resins. Dent
Mater 2003; 19: 12-18.

7. Turkun LS, Turkun M. The effect of one-step polishing system on the surface roughness of three esthetic resin composite materials. Oper Dent 2004; 29: 203-211.

8. Yalcin F, Korkmaz Y, Baseren M. The effect of two different polishing techniques on microleakage of new composites in Class V restorations. J Contemp Dent Pract 2006;7:18-25.

9. Alawjali SS, Lui JL. Effect of one-step polishing system on the color stability of nanocomposites. J Dent 2013;41 Suppl 3: e53-61.

10. Ono M ve ark. Surface properties of resin composite materials relative to biofilm formation. Dent Mater J 2007; 26: 613-622.

11. Guler AU, Guler E, Yucel AC, Ertas E. Effects of polishing procedures on color stability of composite resins. J Appl Oral Sci 2009; 17: 108-112.

12. Da Costa J, Ferracane J, Paravina RD, Mazur RF, Roeder $L$. The effect of different polishing systems on surface roughness and gloss of various resin composites. J Esthet Restor Dent 2007; 19: 214-224; discussion 25-6.

13. Korkmaz Y, Ozel E, Attar N, Aksoy G. The influence of one-step polishing systems on the surface roughness and microhardness of nanocomposites. Oper Dent 2008; 33 : 44-50

14. Baseren M. Surface roughness of nanofill and nanohybrid composite resin and ormocer-based tooth-colored restorative materials after several finishing and polishing procedures. J Biomater Appl 2004; 19: 121-134.

15. Attar N. The effect of finishing and polishing procedures on the surface roughness of composite resin materials. $J$ Contemp Dent Pract 2007; 8: 27-35.

16. Antonson SA, Yazici AR, Kilinc E, Antonson DE, Hardigan PC. Comparison of different finishing/polishing systems on surface roughness and gloss of resin composites. J Dent 2011; 39 Suppl 1: e9-17.

17. Stoddard JW, Johnson GH. An evaluation of polishing agents for composite resins. J Prosthet Dent 1991; 65: 491 495.

18. Borges AB, Marsilio AL, Pagani C, Rodrigues JR. Surface 
roughness of packable composite resins polished with various systems. J Esthet Restor Dent 2004; 16: $42-47$

19. Roeder LB, Tate WH, Powers JM. Effect of finishing and polishing procedures on the surface roughness of packable composites. Oper Dent 2000; 25: 534-543.

20. Yap AU, Lye KW, Sau CW. Surface characteristics of tooth-colored restoratives polished utilizing different polishing systems. Oper Dent 1997; 22: 260-265.

21. Setcos JC, Tarim B, Suzuki S. Surface finish produced on resin composites by new polishing systems. Quintessence Int 1999; 30: 169-173.

22. Ozgunaltay G, Yazici AR, Gorucu J. Effect of finishing and polishing procedures on the surface roughness of new tooth-coloured restoratives. J Oral Rehabil 2003; 30: 218224.

23. Barbosa SH, Zanata RL, Navarro MF, Nunes OB. Effect of different finishing and polishing techniques on the surface roughness of microfilled, hybrid and packable composite resins. Braz Dent J 2005; 16: 39-44.

24. Lu H, Roeder LB, Lei L, Powers JM. Effect of surface roughness on stain resistance of dental resin composites. J Esthet Restor Dent 2005; 17: 102-8

25. Bashetty K, Joshi S. The effect of one-step and multistep polishing systems on surface texture of two different resin composites. J Conserv Dent 2010; 13: 34-38.

26. Ozel E, Korkmaz Y, Attar N, Karabulut E. Effect of onestep polishing systems on surface roughness of different flowable restorative materials. Dent Mater J 2008; 27: 755764.

27. Yap AU, Yap SH, Teo CK, Ng JJ. Finishing/polishing of composite and compomer restoratives: effectiveness of one-step systems. Oper Dent 2004; 29: 275-279.

28. St-Georges AJ, Bolla M, Fortin D, et al. Surface finish produced on three resin composites by new polishing systems. Oper Dent 2005; 30: 593-597.

29. Özel Y, Çelik, Ç., Karabulut, E. Farklı polisaj sistemlerinin kompozitlerin yüzey pürüzlülüğüne etkisi. SÜ Dişhek Fak Der 2008; 17: 39-43.

30. Erdemir U, Sancakli HS, Yildiz E. The effect of one-step and multi-step polishing systems on the surface roughness and microhardness of novel resin composites. Eur J Dent 2012; 6: 198-205.

31. Koh R, Neiva G, Dennison J, Yaman P. Finishing systems on the final surface roughness of composites. J Contemp Dent Pract 2008; 9: 138-145.

32. Uctasli MB ve ark. The effect of different finishing and polishing systems on the surface roughness of different composite restorative materials. J Contemp Dent Pract 2007; 8: 89-96.

33. Gonulol N, Yilmaz F. The effects of finishing and polishing techniques on surface roughness and color stability of nanocomposites. J Dent 2012; 40 Suppl 2: e64-70.

34. Lepri CP, Palma-Dibb RG. Surface roughness and color change of a composite: influence of beverages and brushing. Dent Mater J 2012; 31: 689-696.

35. Ergucu Z, Turkun LS. Surface roughness of novel resin composites polished with one-step systems. Oper Dent 2007; 32: 185-192.

36. Chung $\mathrm{KH}$. Effects of finishing and polishing procedures on the surface texture of resin composites. Dent Mater 1994; 10: 325-330.

37. Kaplan BA, Goldstein GR, Vijayaraghavan TV, Nelson IK. The effect of three polishing systems on the surface roughness of four hybrid composites: a profilometric and scanning electron microscopy study. J Prosthet Dent 1996; 76: 34-38.

38. Endo T, Finger WJ, Kanehira M, Utterodt A, Komatsu M. Surface texture and roughness of polished nanofill and nanohybrid resin composites. Dent Mater J 2010; 29: 213223.

39. Marghalani HY. Effect of finishing/polishing systems on the surface roughness of novel posterior composites. J Esthet Restor Dent 2010; 22: 127-138.

40. Kakaboura A, Fragouli M, Rahiotis C, Silikas N. Evaluation of surface characteristics of dental composites using profilometry, scanning electron, atomic force microscopy and gloss-meter. J Mater Sci Mater Med 2007; 18: 155-163. 